\title{
Not all dementia is Alzheimer: Dementia with Lewy bodies
}

David Knopman, MD; and Janet Jankowiak, MD

Dementia with Lewy bodies (DLB) can be a tricky disease to understand. In simple terms, DLB is a combination of dementia and Parkinson disease (PD). Dementia refers to a problem with normal daily activities due to problems thinking (see "About dementia and similar conditions" on the following page). The most common problems in dementia are short-term memory loss, lack of drive, and slowed thinking. PD typically includes a tremor (shaking) that is present at rest, slowed movements, and loss of facial expression. People with PD have a characteristic walk: they are stooped over, don't swing their arms normally, take small, shuffling steps, and may lose their balance.

\section{What are Lewy bodies?}

Lewy bodies are seen in brain tissue viewed under the microscope. They are known to be the hallmark of PD. They usually occur in a part of the brain known as the substantia nigra deep within the brain, but also sometimes occur in other brain locations. The significance of Lewy bodies found outside the substantia nigra was not appreciated until recently. Also, Lewy bodies in the brain were previously thought to indicate PD, and nothing else.

About 20 years ago, neurologists and neuropathologists (scientists who study the structure of the brain) came to recognize that Lewy bodies were also found in patients with a dementing illness that often had been called Alzheimer disease (AD) when the patient was first diagnosed. New insights led to more careful observations about the distinctive features of DLB. In 1996, Dr. Ian McKeith and coworkers brought together a group of scientists interested in defining DLB. ${ }^{1}$ Their first publication identified some core features of what they called DLB. These included dementia, parkinsonism (features that look like PD), marked variations in arousal, hallucinations, and extreme sensitivity to antipsychotics (medications used to treat hallucinations and psychosis).

From that starting point in 1996, the joint efforts of neurologists, psychiatrists, sleep physicians, and neuropathologists have led to a blossoming of understanding of DLB. Much progress has been made in characterizing and treating DLB. DLB is actually more than just dementia and PD. In this issue of Neurology, Dr. McKeith and an international group of clinicians and researchers report on their efforts to better define the clinical and anatomic features of the disease. ${ }^{2}$ They also outline some of the treatment choices for the disorder.

\section{What are the unusual features of $D L B$ ?}

The thinking problems in DLB include problems with memory just like in $A D$, but the memory difficulties in DLB are milder than in AD. In many patients with DLB, attention and concentration are somewhat more affected compared to AD.

The problems with movement in DLB can be very much like typical PD. Slowness of movement and thinking is quite common in DLB. Walking and balance problems are very prominent in DLB, and falls are common. Tremor is less common in DLB than in typical PD.

DLB has some other unique features as well. From day to day, many patients with DLB experience marked fluctuations in how alert they are. These patients may seem very confused one day and very sharp the next. Many patients with DLB experience dramatic, detailed visual hallucinations. These resemble dreams in their vividness and their detachment from reality. DLB patients also experience a peculiar sleep disorder called REM sleep behavior disorder, known as RBD. RBD can occur years before the diagnosis of DLB is made. In RBD, patients experience frightening dreams. They thrash about in their sleep, may strike their bed-partners, and may fall out of bed.

Patients with DLB also exhibit a remarkable sensitivity to many, but not all, antipsychotic medications such as haloperidol or risperidone. Because DLB patients either have $\mathrm{PD}$ or are on the verge of it, they are very sensitive to the Parkinsoncausing side effects of this class of medication.

There are many aspects to DLB that make it challenging to manage. Fortunately, there are treatments for the thinking and movement problems, the hallucinations, and the sleep disorder. Developing an integrated approach to these problems can be tricky.

Treatment for one of the features of the disease can worsen others. With the increased understanding of DLB provided by McKeith and coworkers, physicians have much more to offer patients with DLB and their families.

\section{References}

1. McKeith IG, Galasko D, Kosaka K, et al. Consensus guidelines for the clinical and pathologic diagnosis of dementia with Lewy bodies (DLB): report of the consortium on DLB international workshop. Neurology 1996;47:1113-1124.

2. McKeith IG, Dickson DW, Lowe J, et al. Diagnosis and management of dementia with Lewy bodies: third report of the DLB consortium. Neurology 2005; 65:1863-1872. 


\section{What is dementia?}

Dementia is the diagnosis (label) given when a person has problems thinking and remembering, which interfere with his/her normal daily functioning.

When a neurologist first sees a person for possible dementia, he/she first considers whether there could be some other condition causing the problems. Three other conditions that can be confused with dementia are delirium, depression, and mild cognitive impairment.

\section{What is delirium?}

When a person rapidly develops confusion and changes in thinking, neurologists call this delirium. Examples are intoxication with alcohol or other drugs and infections involving the brain. Fortunately, this condition generally resolves when the underlying cause is fixed or resolves. In contrast, dementia usually develops much more slowly and the underlying cause may not be obvious or correctable. Usually it is possible to separate dementia from delirium. However, some patients with dementia with Lewy bodies (DLB) experience fluctuations and periods of heightened confusion that can look like delirium.

\section{What is depression?}

The key feature of depression is the obvious dramatic appearance of sadness and low mood. Depression is often accompanied by poor

concentration, poor attention span, and poor memory, which may suggest dementia. However, people with depression often complain constantly about their memory. On the other hand, people with dementia generally do not complain about problems with memory because they are unaware of the problem. In the early stages, people with dementia even may make excuses for why they forgot something. Patients with DLB, and people with other types of dementia, may have dementia and depression at the same time.

\section{What is mild cognitive impairment?}

Mild cognitive impairment is also a disorder of thinking, but daily functioning is still preserved. In contrast, patients with dementia have problems with their normal daily activities. Some people with the features of DLB, such as problems with balance and sleep, may not have dementia, but instead may have mild cognitive impairment. Neurologists believe that mild cognitive impairment is a stage just before the development of true dementia.

\section{What is Alzheimer disease?}

Alzheimer disease (AD) is the most common form of dementia. AD makes up about $60 \%$ to $80 \%$ of all dementia. About $30 \%$ of patients with AD go on to develop PD. Many of these people will have DLB. Under the microscope, it is common to see changes in the brain, both of $A D$ and DLB in the same person.

\section{What else can cause dementia?}

Stroke can cause dementia. Vascular dementia is the name given when strokes are the main cause of dementia. Strokes can also cause problems with walking, balance, vision, and speech.

Some medications can affect thinking and memory. Examples are medications to treat sleep problems and anxiety. These drugs are easily capable of making a person slow down mentally, and therefore activities that require full alertness (such as driving) should be avoided while on these medications. Medications for pain often have similar effects. Some medications such as the antipsychotic drugs (used to treat hallucinations and psychotic behavior) can cause many of the symptoms of PD (especially slowed movements and sometimes slowed thinking). Because every person is unique, it is not always possible to predict what side effects may occur.

A wide variety of abnormal levels of substances carried in the blood, including nutrients and hormones, can cause delirium or dementia. The most common disorders that cause delirium are abnormal levels of sodium and calcium, serious loss of kidney or liver function, major declines in thyroid hormone levels, and severe decreases in levels of vitamin $B_{12}$ in the blood. Because delirium and dementia can be difficult to tell apart at times, neurologists check for these abnormalities. They are all easy to diagnose with inexpensive blood tests and are often treatable.

A condition known as normal pressure hydrocephalus (NPH) can mimic DLB. $\mathrm{NPH}$ is a very rare condition that causes a distinctive problem in walking, loss of bladder control, and dementia. A CT scan or MRI of the brain of a patient with NPH shows dramatic enlargement of the brain ventricles (the fluid-filled inner cavities of the brain) out of proportion to the apparent shrinkage of the brain itself. In appropriate patients, a tube can be placed surgically inside the brain ventricles to drain the excess CSF. In the right patient, ventriculoperitoneal shunting, as the procedure is called, can bring considerable improvement in walking, bladder control, thinking, and memory.

A head injury, even relatively mild, may cause problems that mimic dementia. A fall or bump to the head may cause blood clots to form between the brain and the inner side of the skull (called a subdural hematoma). These are particularly common in older people who fall or bump their head and do not think the injury is serious or even forget about it. Often there may be rebleeding within the subdural hematoma with progressive or more rapid loss of mental function. Removal of the subdural hematoma by surgery can cure the symptoms of memory loss and in other cases can be life-saving.

There are many conditions that can mimic dementia and many different causes of dementia. It is important to seek medical help early when problems with thinking occur, because treatments are available.

\section{For more information}

American Academy of Neurology

Foundation

www.thebrainmatters.org

Alzheimer's Association

www.alz.org

Lewy Body Dementia Association, Inc. www.lewybodydementia.org 


\section{Neurology}

\section{Not all dementia is Alzheimer: Dementia with Lewy bodies David Knopman and Janet Jankowiak \\ Neurology 2005;65;E26-E27 \\ DOI 10.1212/01.wnl.0000194990.62816.4c}

\section{This information is current as of December 27, 2005}

\author{
Updated Information \& \\ Services \\ Supplementary Material

\section{References} \\ Permissions \& Licensing \\ Reprints
}

including high resolution figures, can be found at: http://n.neurology.org/content/65/12/E26.full

Supplementary material can be found at: http://n.neurology.org/content/suppl/2012/03/20/65.12.E26.DC1

This article cites 2 articles, 2 of which you can access for free at: http://n.neurology.org/content/65/12/E26.full\#ref-list-1

Information about reproducing this article in parts (figures,tables) or in its entirety can be found online at:

http://www.neurology.org/about/about_the_journal\#permissions

Information about ordering reprints can be found online:

http://n.neurology.org/subscribers/advertise

Neurology ${ }^{\circledR}$ is the official journal of the American Academy of Neurology. Published continuously since 1951, it is now a weekly with 48 issues per year. Copyright . All rights reserved. Print ISSN: 0028-3878. Online ISSN: 1526-632X.

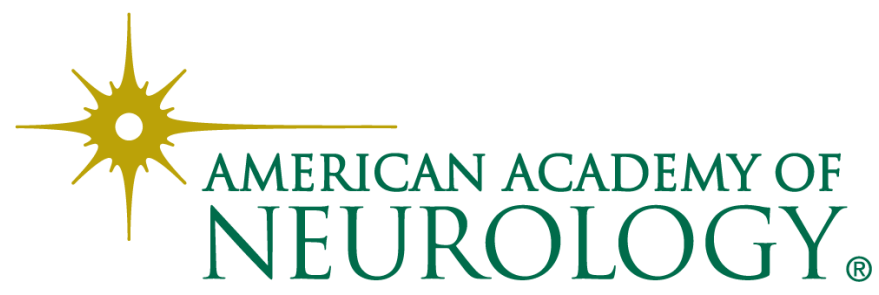

